\title{
Enseigner l'occitan au XXIe siècle. Défis et enjeux
}

\section{Marie-Jeanne Verny}

\section{CpenEdition}

Journals

Édition électronique

URL : http://journals.openedition.org/trema/962

DOI : 10.4000/trema.962

ISSN : 2107-0997

Éditeur

Faculté d'Éducation de l'université de Montpellier

\section{Édition imprimée}

Date de publication : 1 septembre 2009

Pagination : 69-83

ISSN : $1167-315 X$

\section{Référence électronique}

Marie-Jeanne Verny, "Enseigner l'occitan au XXle siècle. Défis et enjeux », Tréma [En ligne], 31 | 2009, mis en ligne le 01 septembre 2011, consulté le 10 décembre 2020. URL : http:// journals.openedition.org/trema/962 ; DOI : https://doi.org/10.4000/trema.962

Ce document a été généré automatiquement le 10 décembre 2020.

Trema 


\section{Enseigner l'occitan au XXIe siècle. Défis et enjeux}

Marie-Jeanne Verny

I. L'espace occitan, un espace linguistique complexe

I. 1 Quelques jalons historiques d'abord' 
1 Au Moyen âge, la langue occitane connaît diverses dénominations au niveau littéraire (romans, proensals, lemosi). Tout comme pour le français, souvent désigné lui aussi par roman, il s'agit avant tout de distinguer du latin même la langue qui en est issue. Le nom de lenga d'òc apparaît plus tard, entre la fin du XIIIe et le début du XIVe siècle à la fois dans l'usage administratif du Royaume et sous la plume de Dante qui distingue langue d'oc, langue d'oil et langue de si à partir de la particule affirmative "oui » employée respectivement par les diverses langues (òc, oui, sì).

2 C'est d'abord bien évidemment la langue quotidienne de la population française méridionale qui permet aujourd'hui encore de définir l'Occitanie ${ }^{2}$, mais c'est aussi une langue écrite: un écrit littéraire de

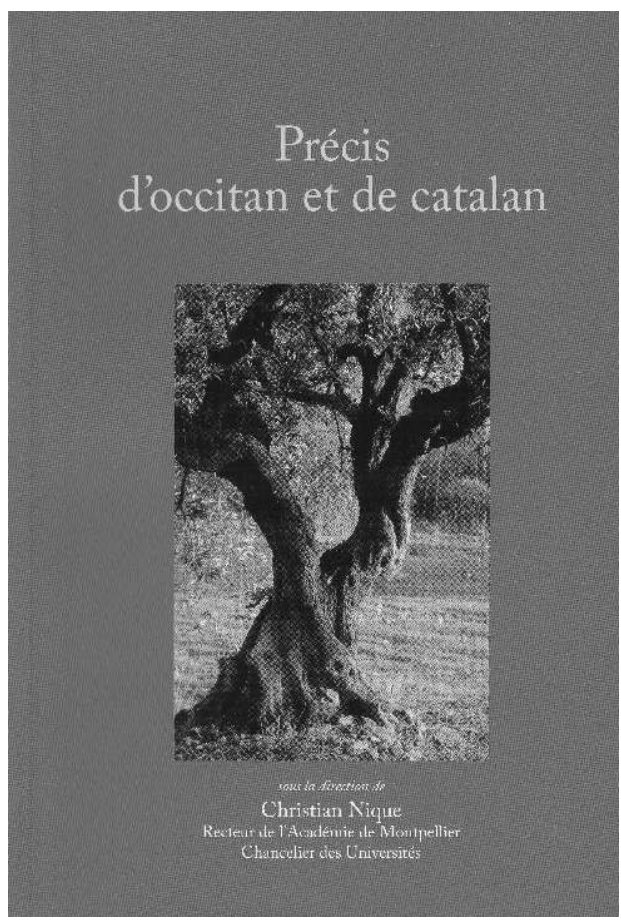
prestige (troubadours), un écrit littéraire plus fonctionnel (manuels didactiques, chroniques urbaines, textes religieux), un écrit social (chartes, administration, correspondances, actes notariés, etc.).

3 L'espace linguistique occitan ne peut en aucune manière être corrélé, dans sa globalité, à un espace administratif, politique ou historique qui ferait consensus.

4 La seule réalité qui fasse l'unanimité - à quelques nuances près ${ }^{3}$ - est celle d'un ensemble linguistique dont on admet qu'il est subdivisé en grandes formes dialectales. Les deux mouvements majoritaires que sont le Félibrige ${ }^{4}$ d'un côté, le mouvement occitan de l'autre, s'accordent sur cette unité linguistique appelée pour le premier «langue d'oc », pour le second « occitan ».

5 Cependant, ces deux mouvements historiques, le premier né au XIXe siècle, le deuxième au XXe, ont abouti à la mise en circulation de deux graphies de l'occitan, l'une appelée «mistralienne», qui suit la tradition ouverte par Mirèio, le chef-d'œuvre mistralien, l'autre appelée " occitane » ou «classique », codifiée par rapport à deux références : la graphie des Troubadours et la normalisation catalane du XXe siècle.

6 L'aura de MISTRAL, son génie littéraire a, par ailleurs, en Provence, entraîné l'adhésion d'une grande partie de la population à l'appellation de "provençal », que Mistral entendait parfois au sens général de «langue d'oc », et qui pour certains, se réfère à l'espace provençal stricto sensu.

$7 \mathrm{Si}$, dans le cadre universitaire notamment, ainsi que dans celui des concours de recrutement des enseignants, les deux graphies sont acceptées, c'est à la graphie dite «occitane» ou «classique » que je me référerai désormais, comme je me situerai, globalement, dans le cadre des analyses occitanistes.

8 La codification de l'occitan écrit obéit généralement à une double volonté :

- des principes communs à l'ensemble de l'espace

- une adaptation à chacun des grands ensembles dialectaux. 
La carte qui suit, extraite du Précis d'occitan et catalan, permet de voir à la fois l'extension de l'espace linguistique occitan, situé essentiellement dans l'Etat français, avec quelques territoires côté espagnol et italien. Elle montre également les grandes variétés dialectales de l'occitan communément reconnues.

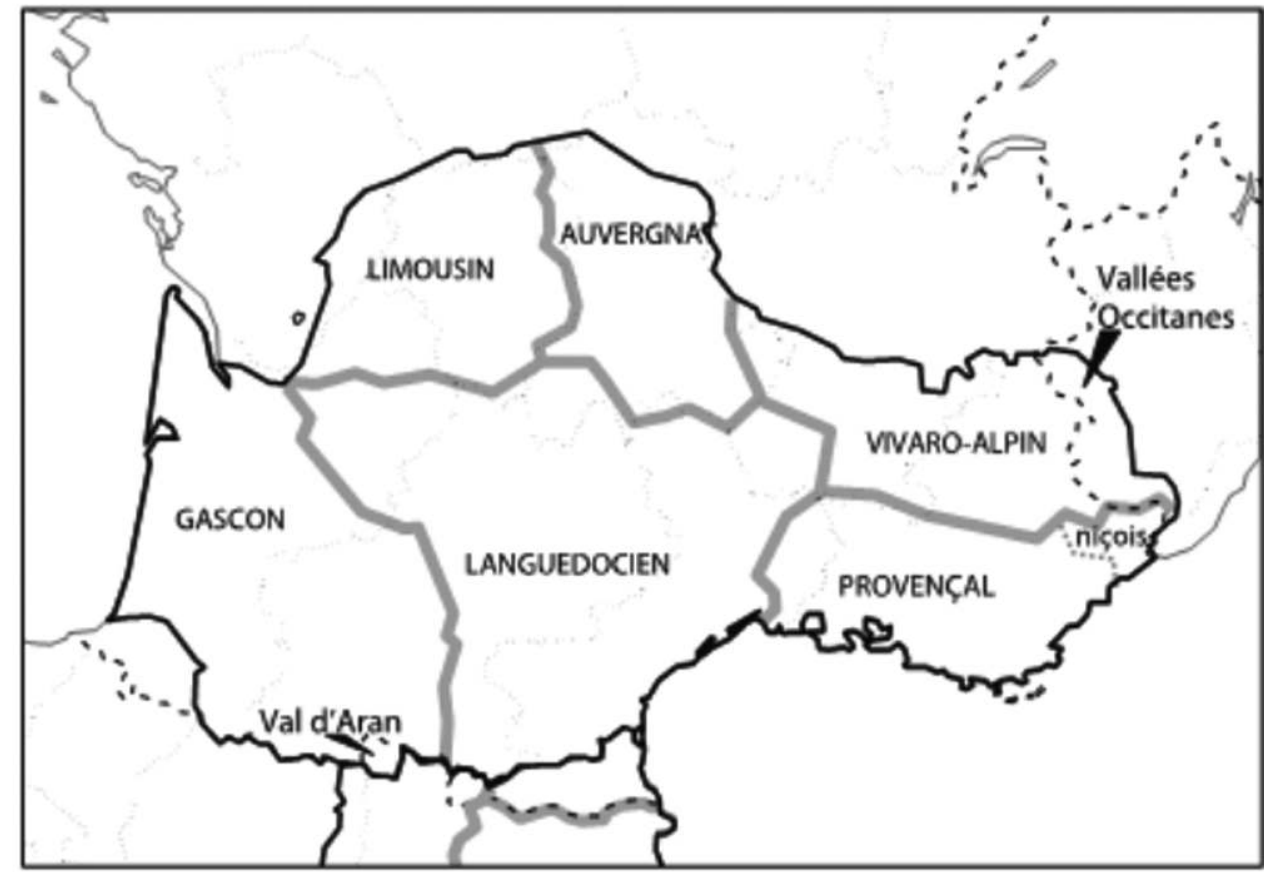

Carte linguistique de l'occitan

\section{2. Une richesse culturelle et linguistique malgré des blocages persistants}

L'étendue de l'espace occitan est certes un atout, qui fait de la langue d'oc la première langue régionale de France par rapport au nombre d'élèves concernés. Cette étendue donne lieu à une richesse culturelle sans commune mesure, dans le domaine de la littérature, bien connu depuis les troubadours, mais aussi dans celui de la musique et de la chanson où on observe depuis une quinzaine d'années une explosion créatrice.

Cependant, cette étendue a pour corollaire négatif la difficulté, pour les militants, de trouver des interlocuteurs institutionnels qui répondent de cet espace sur un plan politico - administratif. L'espace occitan est en effet éclaté entre plusieurs régions administratives et force est de constater qu'en dehors d'initiatives ponctuelles, les responsables de ces régions ne sont guère portés à la collaboration interrégionale qui permettrait de donner une existence effective, même sur des projets limités, à cet espace occitan. Force est aussi de constater que, malgré un investissement général en croissance sensible, l'engagement financier de ces diverses régions en faveur de la langue et de la culture occitanes est très inégal ${ }^{5}$.

Par ailleurs, en l'absence d'une reconnaissance institutionnelle de la langue, en l'absence d'un territoire de légitimation historique, géographique, administratif, ou même simplement symbolique où elle soit reconnue de manière incontestable, la tendance des locuteurs naturels est à la non reconnaissance de la cohérence de cet ensemble linguistique. 
On aboutit donc à une situation que résume fort bien Christian NIQUE (2006) :

« Les locuteurs naturels de l'occitan sont parfois les premiers à faire une différence entre ce qu'ils nomment leur «patois » local et «l'occitan », qu'ils voient comme une langue lointaine et académique. En fait, le concept d'occitan englobe aussi bien les parlers locaux que la variété dite standard. Mais la grande question pour les Occitans est celle de la conscience linguistique. La plupart des locuteurs ont été pendant des siècles et sont encore des «Occitans sans le savoir, Occitans sens o saber » comme dit l'écrivain Jean Boudou $»^{6}$.

Le premier défi est celui de la normalisation de la langue. Ce mot, il faut le préciser, ne s'entend pas en France avec le sens qu'il a en Espagne, sous l'influence de la sociolinguistique catalane, de mise en circulation institutionnelle et sociétale normale. Le rapport de la France à ses langues est tel que nul, même les plus actifs des militants, n'envisage, dans un avenir proche, une co-officialité, à côté du français, des autres langues de France. Par normalisation, nous entendons donc l'élaboration et la mise en circulation d'une norme graphique qui tienne compte de la diversité dialectale.

Dans le Précis d'occitan et de catalan (NIQUE, 2006), ce choix est présenté simplement :

«L'occitan n'a ni statut ni standard, même s'il a connu des tentatives de normalisation au cours de son histoire. Le fait qu'il ait toujours été écrit, sans interruption depuis le Moyen âge, a entraîné l'établissement - certes plus ou moins reconnu - de codes et de règles : à toutes les époques ont été édités des grammaires, des dictionnaires, des études de la langue. Actuellement, les linguistes continuent à élaborer la standardisation et tous s'accordent sur la nécessité de respecter les variétés dialectales, tout en cherchant des solutions convergentes. Le standard est nécessaire pour que l'occitan devienne accessible au grand public. Mais il se nourrit des parlers locaux, de l'occitan hérité qui est le seul légitime. » (p. 72)

\section{Enseigner l'occitan au XXle siècle}

\section{1 Un combat toujours réitéré}

La réflexion sur la codification de l'occitan a été faite en étroite corrélation avec les luttes pour imposer un enseignement de la langue. Ces luttes sont anciennes, comme le montre l'ouvrage récent de Philippe MARTEL, L'école française et l'occitan - Le Sourd et le bègue ${ }^{7}$.

17 Le sourd, c'est la France, le bègue, c'est le militant de l'enseignement de l'occitan qui depuis le XIXe siècle, inlassablement, répète les mêmes revendications, suscite des propositions de loi dont la plupart n'arrivent même pas en discussion en séance de l'Assemblée Nationale. Pour ne parler que des 60 dernières années, ce sont souvent les mêmes hommes et femmes qui, au sein de l'Institut d'Études Occitanes (IEO, créé en 1945, à l'issue de la guerre et dans l'aura de résistants de plusieurs de ses fondateurs), bâtissaient des outils pédagogiques, participaient aux réflexions linguistiques, formaient des groupes de pression pour que le législateur organise l'enseignement de la langue.

Ces groupes associaient souvent des militants des autres domaines linguistiques, Bretagne notamment. C'est ce double engagement qui caractérise les acteurs de l'enseignement public de l'occitan :

- un combat militant, en liaison avec des partenaires multiples (syndicats d'enseignants, fédérations de parents d'élèves, associations de défense des autres langues de France, élus des différentes collectivités territoriales, représentants de l'État). 
- une réflexion pédagogique intense pour repenser l'apprentissage de la langue en tenant compte aussi bien du contexte sociolinguistique de celle-ci que de sa spécificité de langue pluridialectale, sans oublier une attention particulière portée aux recherches générales en didactique des langues.

Dans une période récente, un manuel destiné aux lycéens, Òc-Ben (littéralement, Oui, bien sûr), donnait un bon exemple de prise en compte pédagogique de la diversité dialectale de l'espace occitan dans son unité fondamentale. Chaque leçon est transcrite dans quatre des grands dialectes de l'occitan, puis une page unique intitulée "Travèrsas", montre l'articulation des dialectes entre eux, leurs constances et leurs différences, tout en donnant des clés pour passer d'un dialecte à l'autre, au moins dans un cadre de compétence passive.

\section{2 Principes et réalités}

20 Enseigner l'occitan, c'est théoriquement possible à tous les niveaux, selon des modalités variables, mais le cadre juridique est très fragile et les inégalités très importantes d'un point à un autre du territoire. À ce sujet, on note que les zones dans lesquelles la langue est la plus vivante et compte le plus de locuteurs naturels (les zones rurales et les zones de montagne du Nord de l'espace - Auvergne, Limousin, notamment) sont celles où l'enseignement est le moins organisé, où existent peu de postes de personnels qualifiés. Une explication réside dans le sentiment d'autodépréciation dont sont encore prisonniers ces locuteurs naturels, qui les freine dans leur exigence d'enseignement de leur langue, quand elle ne sert pas tout bonnement d'obstacle.

\section{3 Les avancées les plus récentes}

21 Si l'inscription juridique des langues régionales dans l'enseignement date de la Loi DEIXONNE (1951), il faut souligner les avancées les plus récentes depuis cette date :

- création de classes bilingues publiques en 1982

- création du CAPES d'occitan-langue d'oc en 1992. Ce concours, pour le domaine occitan, est bivalent. Les candidats doivent passer aussi des épreuves (le quart du total environ) dans une autre discipline, à choisir entre anglais, espagnol, histoiregéographie, lettres modernes.

- création du concours spécifique de professeurs des écoles de et en langues régionales en 2002. Noter que ce concours n'est pas organisé dans toutes les académies.

- dans un contexte plus global, l'inscription des langues régionales dans la Constitution de la République date de juillet 2008 : « Les langues régionales appartiennent au patrimoine de la France ». Certes, cette phrase figure dans l'article 75 , qui concerne plutôt les collectivités territoriales. Cette inscription avait été précédée au début des années 2000, par la création, au sein du Ministère de la Culture de la Direction Générale à la Langue Française et aux Langues de France ( http://www.dglflf.culture.gouv.fr/), et elle est actuellement suivie par l'annonce d'une Loi relative aux langues régionales. 


\section{4. La formation à l'Université}

L'occitan est enseigné dans le monde entier et en France, en particulier à Aix, Bordeaux, Clermont-Ferrand, Montpellier, Nice, Pau, Paris-Sorbonne, Toulouse.

Pour prendre l'exemple de Montpellier III, Université Paul-Valéry, le département d'occitan organise des enseignements pour tous les étudiants, débutants ou non :

- occitan pour non-spécialistes : cours de langue vivante et de civilisation.

- licence spécialisée : formation complète en langue et culture occitanes. Cette licence spécialisée peut être monodisciplinaire (occitan seul avec quelques modules optionnels selon le schéma des formations de licence) ou bidisciplinaire (licence occitan / lettres, ou occitan/espagnol, ou occitan / histoire - géographie, ou occitan / anglais, ou occitan /espagnol.

- Tous ces enseignements sont accessibles à distance (par internet).

- préparation aux concours, en liaison avec l'IUFM de Montpellier : CAPES et CRPE (concours de recrutement des professeurs des écoles) spécial langue régionale.

- Master 1 et 2 LCER (langues cultures étrangères et régionales), parcours occitan

- Doctorat de langues romanes mention occitan ...

\section{5 Les effectifs ${ }^{8}$}

Effectifs des élèves répartis par académie et niveau d'enseignement - Année scolaire 2003-2004

\begin{tabular}{|l|c|c|c|c|}
\hline \multicolumn{1}{|c|}{ Académie } & Nb d'élèves Ecole & $\begin{array}{c}\text { Nb d'élèves } \\
\text { Collège }\end{array}$ & Nb d'élèves Lycée & Total \\
\hline Aix-Marseille & 8766 & 2183 & 773 & 11722 \\
\hline Bordeaux & 8456 & 1599 & 391 & 10446 \\
\hline $\begin{array}{l}\text { Clermont- } \\
\text { Ferrand }\end{array}$ & 691 & 145 & 67 & 903 \\
\hline Grenoble & 1413 & 170 & 15 & 1583 \\
\hline Limoges & 104 & 103 & 709 & 13394 \\
\hline Montpellier & 9447 & 3238 & 543 & 4897 \\
\hline Nice & 3491 & 863 & 1357 & 35602 \\
\hline Toulouse & 24929 & 9316 & 3855 & $\mathbf{7 8 7 6 9}$ \\
\hline Total & 57297 & 17617 & & 222 \\
\hline
\end{tabular}

Source : Enquête Desco (Direction de l'Enseignement Scol aire - Ministère de l'Éducation Nationale) Mars 2004

À côté du secteur public, existent des écoles associatives "calandretas », qui regroupent une cinquantaine d'établissements, dont deux collèges, avec 2500 élèves. Les enfants y pratiquent en complémentarité deux langues vivantes: le français et l'occitan. La méthode pratiquée est celle de l'immersion linguistique précoce. 


\section{6. Des avancées dans le domaine de la réflexion pédagogique et didactique}

L'institutionnalisation de la fonction des enseignants de langue régionale (CAPES d'occitan-langue d'oc, et CRPE spécifique langues régionales), a eu pour corollaire un développement de la réflexion sur la formation des maîtres, des actions de mise en œuvre de celle-ci et pour conséquence, la création d'outils pédagogiques.

\section{6. 1. La formation des maîtres}

26 Les IUFM concernés ont, à des degrés divers, créé des formations en occitan. Cela va de quelques heures annuelles de formation linguistique et didactique ouverte optionnellement à tous les candidats des concours du premier degré, jusqu'à une formation approfondie pour les maîtres à statut particulier, titulaires du CAPES ou du CRPE spécifique.

Enseigner une langue, cela s'apprend. Cela suppose dans un premier temps une exigence dans ses propres compétences de locuteur, mais aussi de scripteur de la langue.

Cela suppose, bien entendu, une maîtrise d'un savoir universitaire au moins égal à la licence dans des disciplines aussi différentes que la pratique orale et écrite de la langue, l'histoire, la civilisation, la linguistique moderne et médiévale, la sociolinguistique, l'histoire de la langue, l'histoire de la littérature, l'analyse des textes littéraires ...

Mais cette maitrise des contenus a pour corollaire une réflexion sur les pratiques de classe. La professionnalisation de l'enseignement de l'occitan a continué le chemin ouvert par les pionniers des époques militantes (après 1945) qui s'étaient imposé cette exigence de pratiques raisonnées, pensées, réfléchies 9 .

Cette exigence qui accompagnait ces pionniers et servait à légitimer leur engagement a donc entraîné, avec la création de ces statuts d'enseignants d'occitan acquis avec le CAPES et le CRPE, la prise en charge par l'institution de la formation des maitres, alors que se poursuivent les stages pédagogiques spécifiques associatifs.

Parmi les éléments de formation, on peut énumérer quelques points :

- comment enseigne-t-on une langue ? Cette question, qui semble aller de soi, mérite cependant d'être posée. Apprendre une langue, dans le cadre scolaire, cela passe par d'autres moyens que l'apprentissage spontané par la fréquentation des locuteurs naturels, fréquentation difficile, en domaine occitan par la raréfaction de ceux-ci, mais aussi à cause des blocages induits par le complexe diglossique ${ }^{10}$.

- comment construit-on un processus d'apprentissage? La réflexion actuelle repose notamment sur le CECRL (Cadre européen commun de référence pour les langues, défini sur le site du Ministère de l'Éducation Nationale : http://eduscol.education.fr/ D0067/cecrl.htm)

- comment évalue-t-on les savoirs acquis par les élèves ?

- quels outils pédagogiques utiliser et comment les utiliser au mieux?

- quelles pratiques interdisciplinaires sont-elles utiles? Sur ce point plusieurs réflexions sont menées, autour de quelques pôles forts que sont par exemple les parcours romans (travaux bâtis à partir de la comparaison entre les langues romanes), les travaux interdisciplinaires entrant dans le cadre de projets 
pédagogiques d'établissement (occitan / lettres, occitan / histoire - géographie, occitan / arts plastiques, occitan / sport ...)

- comment gérer l'hétérogénéité des élèves?

- la langue occitane une dans sa diversité : quelles conséquences pédagogiques? Quelle aide apporter à un jeune enseignant affecté dans une zone géographique dont le parler naturel n'est pas le sien?

- l'enseignant d'occitan et sa discipline, entre reconnaissance institutionnelle et blocages administratifs ou socio - psychologiques.

\section{6. 2. La création d'outils pédagogiques}

Il s'agit, là encore, d'une constante de la pédagogie occitane. Les plus grands penseurs de l'occitanisme (Charles CAMPROUX ${ }^{11}$ ou Robert LAFONT ${ }^{12}$ par exemple) ont aidé à la construction de ces outils. Cependant, comme pour la formation des maîtres, l'institution a, peu à peu, pris le relais, soutenue par une grande implication bénévole des concepteurs de méthodes et manuels et des animateurs de revues. Soutenue aussi, depuis quelques années, par les collectivités locales, qui contribuent fortement au financement.

Ces outils pédagogiques sont donc pris en charge par les CRDP (Centres Régionaux de Documentation Pédagogique), sous l'égide du CNDP (national). On en trouvera un catalogue complet sur le site du CNDP / SCEREN : http://www.sceren.com/cyber-librairiecndp.asp? $=$ occitan $\&$ cat $=86310$

Trois CRDP sont particulièrement actifs en la matière: Bordeaux (http://crdp.acbordeaux.fr/capoc/), Montpellier (http://www.crdp-montpellier.fr/languesregionales/) et Toulouse-Midi-Pyrénées (http://www.crdp-toulouse.fr/?page=recherche\&univers=18\& recherche=occitan).

\section{Quelques exemples de productions pédagogiques}

\section{Albums et livres de lecture}

La nécessité de publier des ouvrages de lecture en occitan s'est imposée d'emblée à tous les chercheurs en pédagogie. L'enseignement d'une langue vivante menacée, difficilement reconnue par la société, passe par la communication aux élèves de productions variées dans cette langue, et notamment de productions modernes et attractives.

$\mathrm{Au}$ CRDP de Montpellier, plusieurs albums ont été édités, avec un accompagnement pédagogique : http://www.crdp-montpellier.fr/languesregionales/occitan/ressources/ sceren/sceren_primaire.html

Deux objectifs sont ainsi atteints : donner à lire, en occitan, de vrais livres, de qualité ; fournir aux maîtres des pistes d'exploitation qu'ils n'ont pas toujours le temps d'élaborer. Pour les albums pour enfants, il faut noter aussi le travail du CapOc, (CRDP de Bordeaux), proposant des versions en plusieurs dialectes de l'occitan, avec des fiches pédagogiques : http://crdp.ac-bordeaux.fr/capoc/produccions.asp

D'autres ouvrages sont plutôt édités à destination des élèves plus âgés, ainsi de Las aventuras de Nasr-Eddin par Yves Rouquette, ouvrage trilingue (occitan, français, 
espagnol), édité par le CRDP Midi-Pyrénées. Au CRDP de Montpellier, c'est la collection «Camin de vida » qui est destinée à des lecteurs confirmés. Cependant, il s'agit cette fois d'éditions originales, bilingues et accompagnées d'un CD où le texte est lu.

C'est aussi la collection "Florilègi occitan ", anthologies thématiques, dont deux volumes sont parus : Bestiari ${ }^{13}$, e Lo Cant de las vilas / Le Chant des villes ${ }^{14}$. Ce dernier volume contient, en plus des textes occitans, un texte dans chacune des langues suivantes: allemand, amazigh, anglais, arabe, breton, catalan, espagnol, grec, italien. La polyphonie du chant des villes est ainsi illustrée.

\section{Manuels}

Beaucoup d'entre eux ont un objectif limité : leçons basiques de langue, recueils de textes avec pistes de travail, tels sont les deux modèles dominants, auxquels on peut ajouter les ouvrages d'initiation à la civilisation, dont le remarquable Chercheurs d'oc ${ }^{15}$, édité par l'académie de Toulouse.

Il s'agit d'un ensemble composé d'un livre et d'un DVD. Le DVD contient 32 séquences filmiques qui font découvrir, du Val d'Aran à l'Auvergne et de l'Aquitaine au LanguedocRoussillon, les diverses facettes de la culture occitane: la langue et son histoire, la littérature d'hier et d'aujourd'hui, la musique, la cuisine et les fêtes. Le livre d'accompagnement propose, pour chacun des petits films, des suggestions d'activités pédagogiques et de nombreux documents complémentaires. "Chercheurs d'Oc » est destiné aux élèves "non - occitanistes ", mais peut être utilisé par les professeurs de langue et culture occitane.

Nous avons déjà évoqué, pour sa prise en compte au moins partielle de la variété dialectale de l'occitan l'ouvrage le plus complet et le plus récent : Òc Ben. Cet ouvrage en deux tomes est ainsi présenté sur le site de l'Agence Régionale pour l'écrit et le livre en Aquitaine (http://arpel.aquitaine.fr/spip.php ?rubrique60). Voir bibliographie.

\section{Une revue pédagogique, Lenga e país d'òc}

Elle est également éditée par le CRDP de Montpellier : http://www.crdp-montpellier.fr/ languesregionales/occitan/ressources/sceren/crdp.html et destinée aux enseignants. Chaque numéro est organisé en trois rubriques :

- Estudis présente des études dans différents domaines de recherche en occitan, littérature, langue, histoire, didactique, sociologie...

- Practicas contient des analyses de pratiques pédagogiques et des réflexions sur les modalités et les objectifs de l'enseignement de l'occitan à l'école, au collège et au lycée.

- Tèxtes fournit des regroupements de textes autour d'une période, d'un thème, d'un auteur, avec des pistes d'exploitation pédagogiques. Dans cette rubrique sont également publiées les épreuves d'examens et concours des différentes académies. 


\section{7. Principales difficultés et blocages}

45 Ces points positifs ayant été énoncés, force est de constater que difficultés et blocages persistent, notamment des disparités dans l'espace français, des disparités dans l'espace occitan, des attaques récurrentes depuis 2002, l'annonce de mesures inquiétantes, un cadre idéologique encore négatif, des blocages politico-économiques.

Deux sortes d'obstacles s'imposent donc aux défenseurs des langues régionales :

- Le contexte d'économies budgétaires de plus en plus criantes.

- Le contexte de dévalorisation (voire d'auto - dévalorisation, en ce qui concerne les locuteurs naturels) des langues régionales. Cette dévalorisation est, d'ailleurs, plus souvent le fruit d'une méconnaissance que d'une hostilité déclarée. Beaucoup d'obstacles tombent lorsque l'on présente à leurs détracteurs (ou à ceux qui les ignorent) les richesses des langues et cultures régionales.

\section{7. 1. Des disparités dans l'espace français}

Plusieurs éléments pourraient être pris en compte pour mettre en évidence ces inégalités, totalement contradictoires avec l'affirmation réitérée de la notion d'égalité républicaine.

La densité des sites d'enseignement bilingues publics en est un. Alors qu'en Corse, par exemple, près d'un élève sur deux a accès à cet enseignement bilingue, la majorité des départements occitans sont totalement dépourvus de sites bilingues.

Si l'on considère les postes ouverts au CAPES (concours d'enseignement du second degré), cette disparité est évidente : 
Postes offerts au CAPES externe 2008 (source Ministère de l'Éducation Nationale) Répartition par section et option des postes offerts:

\begin{tabular}{|l|l|}
\hline Sections / options & Postes offerts \\
\hline Langue corse & 3 \\
\hline Langues régionales : basque & 1 \\
\hline Langues régionales : breton & 2 \\
\hline Langues régionales : catalan & 1 \\
\hline Langues régionales : créole & 4 \\
\hline Langues régionales : occitan-langue d'oc & 4 \\
\hline Tahitien & 1 \\
\hline TOTAL & 16 \\
\hline
\end{tabular}

NB: L'allemand est aussi considéré comme « langue régionale d'Alsace ».

Langues vivantes étrangères: allemand 106

Répartition par département:
\begin{tabular}{|l|l|l|}
\hline \multicolumn{2}{|l|}{ Nombre de départements concernés } & $\begin{array}{l}\text { Nombre de postes attribués par } \\
\text { département }\end{array}$ \\
\hline Langue corse & 2 & 1,5 \\
\hline Langues régionales : basque & $1 / 2$ & 2 \\
\hline Langues régionales : breton & 4 & 0,5 \\
\hline Langues régionales : catalan & 1 & 1 \\
\hline Langues régionales : occitan-langue d'oc & équivalent 30 & 0,13 \\
\hline
\end{tabular}

Récapitulatif des postes mis au concours des CAPES de langues régionales depuis 2000 (source : Bulletin Officiel de l'Éducation Nationale - BOEN)

\begin{tabular}{|l|c|c|c|c|c|c|c|c|c|c|c|}
\hline Langue & 2000 & 2001 & 2002 & 2003 & 2004 & 2005 & 2006 & 2007 & total & $\begin{array}{l}\text { Nombre de } \\
\text { départements } \\
\text { concemés }\end{array}$ & $\begin{array}{l}\text { Nombre moyen } \\
\text { de postes créés } \\
\text { par département }\end{array}$ \\
\hline Basque & 5 & 6 & 6 & 4 & 1 & 1 & 1 & 1 & 25 & 0,5 & 50 \\
\hline Breton & 9 & 11 & 8 & 8 & 2 & 2 & 2 & 2 & 44 & 4 & 11 \\
\hline Catalan & 5 & 6 & 6 & 4 & 1 & 1 & 1 & 1 & 25 & 1 & 25 \\
\hline Corse & 6 & 8 & 9 & 7 & 3 & 3 & 3 & 3 & 42 & 2 & 21 \\
\hline Créole & - & - & 11 & 9 & 4 & 4 & 4 & 4 & 36 & 4 & 9 \\
\hline Occitan & 19 & 22 & 20 & 14 & 4 & 3 & 4 & 4 & 90 & 30 & 3 \\
\hline Total & 44 & 48 & 60 & 46 & 15 & 14 & 15 & 15 & 257 & 41,5 & 6,2 \\
\hline
\end{tabular}

\section{7. 2. Des disparités dans l'espace occitan}

50 Par exemple, il n'existe qu'un poste de professeur certifié d'occitan - langue d'oc en Auvergne (académie de Clermont-Ferrand) ou en Limousin (académie de Limoges). Paradoxalement, il s'agit d'académies où la proportion de locuteurs naturels est la plus importante. Preuve supplémentaire que la connaissance de la langue ne va pas forcément de pair avec la reconnaissance de sa valeur.

51 Pour montrer les disparités internes à l'espace occitan, nous avons choisi de montrer la carte des sites bilingues de l'enseignement public. Elle permet de constater une densité très inégale, une inexistence au Nord et à l'Est et une concentration sur le Centre - ouest de l'espace occitan. 


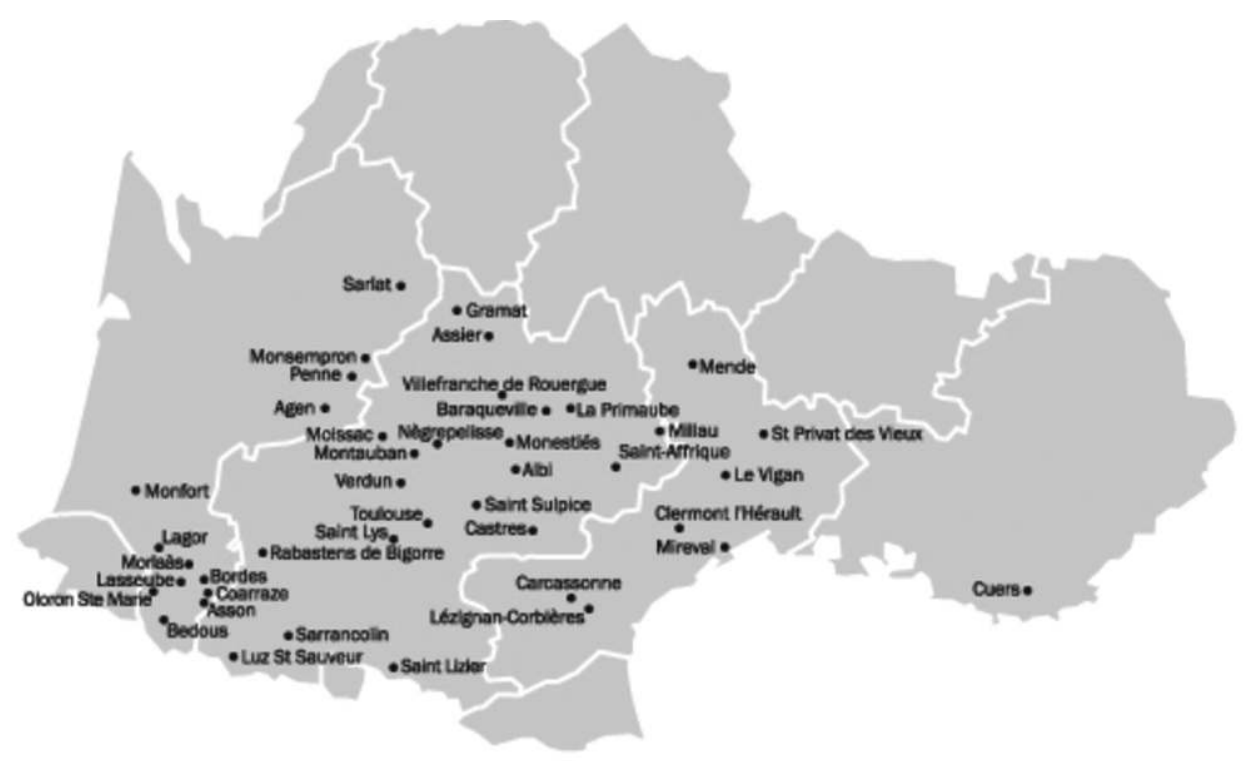

Les sites bilingues publics françals-oceltan

\section{7. 3. Les attaques récentes} . Les langues régionales étant exclues de la certification en langues de fin de troisième, les élèves qui ont pris l'occitan en LV2 ne peuvent passer la certification que dans leur LV1, contrairement à leurs camarades qui ont le choix entre LV1 et LV2 étrangère.

58 - Fin de la prise en compte des langues régionales au concours de professeur des écoles (hors concours spécifiques).

59 - Baisse proportionnelle des coefficients au bac. L'attribution d'un coefficient au baccalauréat de 3 aux langues anciennes, de 2 aux langues régionales rompt le principe d'équité dans les examens, ces deux options linguistiques et culturelles étant tout à fait comparables. 
60 - Menaces sur les postes d'encadrement et d'animation pédagogique. La suppression de ces postes de maîtres formateurs et instituteurs animateurs entraîne une diminution très importante des enseignants qui assurent l'enseignement de l'occitan et en définitive des enfants qui en bénéficient.

61 - Une formule ambivalente dans la récente Loi d'orientation pour l'école dite « loi FILLON » 2005. Le premier alinéa de l'article L. 312-10 du Code de l'Éducation est ainsi rédigé : «Un enseignement de langues et cultures régionales peut être dispensé tout au long de la scolarité par voie de convention entre l'État et les collectivités territoriales où ces langues sont en usage. »(Chapitre III article 20 de la Loi). Comprise dans un sens restrictif, cette phrase pourrait laisser entendre que là où il n'y a pas signature de convention entre l'État et les collectivités locales concernées, l'enseignement de l'occitan pourrait ne pas être organisé. Or, une seule Région occitane a jusqu'ici signé une telle convention : la Région Aquitaine. Partout ailleurs, le blocage des signatures s'apparente, pour les militants de l'enseignement public des langues régionales, à une partie de bras de fer entre les Rectorats, représentants de l'État et les présidents des Régions concernées. Par ailleurs, cette obligation faite par la Loi risque d'accentuer les inégalités entre Régions, voire les replis localistes. La position de la FELCO (Fédération des enseignants de langue et culture d'oc) par rapport à cet article est celle de la prudence devant le risque de désengagement de l'État, qui demeure notre interlocuteur principal, mais aussi la demande réitérée aux collectivités territoriales de s'engager dans l'élaboration et la signature de conventions.

\section{8. Pour terminer sur une note d'espoir : la connaissance de l'occitan, un facteur essentiel pour l'emploi}

62 Le CREO Lengadòc a entrepris en 2008, avec le soutien financier de la Région LanguedocRoussillon, une campagne de sensibilisation auprès des élèves, et, plus largement, de la société civile, sur les emplois offerts à ceux qui ont effectué de solides études d'occitan (au lycée et à l'Université).

63 À la base de ce travail, le constat que les effectifs d'élèves et d'étudiants sont à l'heure actuelle largement insuffisants pour couvrir les besoins.

64 Une enquête réalisée en $2005^{16}$ révélait que les licenciés d'occitan de l'Université Paul Valéry avaient eu les plus grandes facilités à trouver un emploi après leurs études. Au moment de l'enquête, la plupart avaient un travail rémunéré (86\%), 73 \% disaient avoir mis moins d'un an à trouver un emploi après la fin de leurs études, et $63 \%$ affirmaient que leur premier emploi avait un rapport avec leurs études d'occitan, $65 \%$ des enquêtés pensaient que l'occitan les avait aidés à trouver un emploi.

65 La campagne mise en œuvre par le CREO Lengadòc a répertorié les emplois occupés par les jeunes diplômés en occitan. Ils sont très divers : professeurs de l'enseignement public (écoles, collèges, lycées, Universités), regents de calandretas, formateurs pour adultes, employés d'associations culturelles ou de collectivités territoriales, journalistes (presse écrite, radio, télévision), documentalistes-bibliothécaires, chercheurs, webmasters, libraires, cinéastes, chanteurs, comédiens... 


\section{Pour ne pas conclure ...}

Notre double position scientifique et militante nous permet d'affirmer ici que, si les blocages sont encore présents dans cette France où beaucoup ne voudraient voir qu'une seule langue, le français universel, les mentalités bougent. Certes, les moyens - humains et financiers - ne suivent pas la demande sociale. Les jeunes formés sont encore en nombre insuffisant, le lien n'est pas toujours facile à établir entre les néo - locuteurs, ceux pour qui l'occitan est une langue apprise, et les locuteurs naturels.

Cependant, parfois motivée par la nostalgie, souvent portée par des projets d'avenir, l'opinion majoritaire est désormais favorable à la pluralité linguistique du pays. Des jeunes, de plus en plus nombreux, choisissent l'occitan comme langue de création poétique, d'expression musicale ou théâtrale.

Alors oui, nous pensons que les efforts accomplis depuis plus de cinquante ans par la réflexion et l'engagement militant de nos prédécesseurs, au premier rang desquels notre maître Robert LAFONT, n'ont pas été vains.

\section{BIBLIOGRAPHIE}

\section{Sur la langue occitane}

NIQUE, C., dir. (2006). Précis d'occitan et de catalan. Montpellier. Diffusion CNDP.

SUMIEN, D. (2007). La standardisation pluricentrique de l'occitan. Nouvel enjeu sociolinguistique, développement du lexique et de la morphologie, Publications de l'Association Internationale d'Études Occitanes n³, XIII+501 p., Brepols.

\section{Sur les questions d'enseignement}

\subsection{Revues pédagogiques et de réflexion}

- Lenga e país d’òc, revue de réflexion et pratiques pédagogiques éditée par le CRDP de Montpellier. $\mathrm{N}^{\circ} 1: 4 \mathrm{e}$ trimestre 1977 . On retiendra en particulier les $\mathrm{n}^{\circ} 41$ et 42 sur les pionniers de l'enseignement de l'occitan, H. CABANNES-GRACIA, R. CHABBERT, G. GROS, R. LAFONT et P. LAGARDE.

\subsection{Manuels et outils pédagogiques}

Chercheurs d'Oc, à la découverte d'un espace, d'une langue, d'une culture. (2004). Livre et DVD. Toulouse : CRDP de Midi-Pyrénées.

SALLES-LOUSTAU, J. (dir.), TORREILLES, C., ARBOUSSET, G., et al. (2003). Òc-Ben ! première année d'occitan, gascon, lengadocian, lemosin, provençau : livre de l'élève, CRDP d'Aquitaine - Bordeaux, CNDP - Futuroscope (Vienne). Paris : Scéren.

SALLES-LOUSTAU, J.(dir.), TORREILLES, C., ARBOUSSET, G. (2004). Òc-ben! deuxième année d'occitan : livre de l'élève. Bordeaux : CRDP d'Aquitaine. 


\subsection{Ouvrages et thèses de parution récente}

TERRAL, H. (2005). La langue d'oc devant l'école (1789-1951), Puylaurens, IEO/IDECO. Préface de Robert Lafont.

MARTEL, P. (2007). L'école française et l'occitan - Le Sourd et le bègue, Presses Universitaires de la Méditerranée, collection «Études occitanes ", Université Paul-Valéry. Préface de Robert LAFONT.

LIEUTARD, H. \& Verny, M-J. éd. (2008). L'école française et les langues régionales, XIXe - XXe siècle, Presses Universitaires de la Méditerranée, collection « Études occitanes », Université PaulValéry.

Lengas, 65, « L'occitan et le catalan à l'école », numéro coordonné par LIEUTARD, H. et VERNY, MJ. Presses Universitaires de la Méditerranée, Université Paul-Valéry, à paraître en 2009.

COUFFIN, P. (2008). Cinquante ans d'occitan dans l'enseignement public, entre légitimité et légalité (L'enseignement du Languedocien : à l'école, au collège et au Lycée). 682 p. Thèse : Études Oc-citanes : Montpellier III.

LESPOUX, Y. (2009). « Des années 1940 aux années 1990, un demi-siècle de revendication en faveur de l'enseignement de la langue d'oc ». Thèse, Université de Pau et des pays de l'Adour. Soutenance 2009.

\section{Sitographie}

Association internationale d'Études Occitanes, AIEO : http://www.aieo.org/

Association des Professeurs de Langues Vivantes : rubrique « langues de France » : http:// www.aplv-languesmodernes.org/spip.php?rubrique48

Fédération des enseignants de langue et culture d'oc (FELCO) : http://creo-mp.totenoc.org/ felco.html et Centre Régional de l'Enseignement de l'occitan Lengadòc - CREO Lengadòc (association académique fédérée dans la FELCO : www.creo-lengadoc.org

Fédération des Langues Régionales dans l'Enseignement Public : http://www.flarep.com/ Centre National de Documentation Pédagogique, occitan : http://www.sceren.com/cyberlibrairie-cndp.asp?l=occitan\&cat $=86310$

Centres Régionaux de Documentation Pédagogique : Bordeaux http://crdp.ac-bordeaux.fr/ capoc/, Montpellier http://www.crdp-montpellier.fr/languesregionales/ et Toulouse - Midi Pyrénées : http://www.crdp-toulouse.fr/?page=recherche\&univers=18\&recherche=occitan

\section{NOTES}

1. Cf. Précis d'occitan et de catalan, Christian NIQUE, Recteur de l'académie de Montpellier (dir.), 2006.

2. Le terme " Occitanie » ne fait pas référence à un État politique ni à une région historique (il n'y a jamais eu d'État occitan, ni de région occitane au sens d'entité politique) mais sert à définir l'espace géographique dans lequel la population parle la langue occitane, de la même manière que le terme francophonie désigne l'ensemble des pays dans lequel le français a une place administrative, culturelle, voire minoritaire. 
3. Il existe, çà et là, dans l'espace occitan, quelques velléités localistes, refusant de reconnaître l'unité de la langue d'oc, se référant à « des langues d'oc », sur le modèle des langues d'oïl. Les tenants de ces positions sont cependant extrêmement minoritaires, en termes de reconnaissance populaire (même si leur influence est parfois sensible en Provence, Béarn ou Auvergne). L'immense majorité des universitaires, comme l'immense majorité des militants, y compris les tenants actuels de la graphie mistralienne, admet l'unité de la langue d'oc dans sa diversité dialectale.

4. Le Félibrige a été fondé en 1854 par Frédéric MISTRAL.

5. Trois régions sont fortement investies : Aquitaine, Languedoc-Roussillon, Midi-Pyrénées.

6. Précis d'occitan et de catalan, sous la direction de Christian NIQUE, Recteur de l'académie de Montpellier, CRDP Montpellier, 2006, p. 18.

7. Presses Universitaires de la Méditerranée, collection "Études occitanes ", Université PaulValéry, 2007, préface de Robert Lafont. http://www.pulm.fr/l-ecole-francaise-et-l-occitan? var_recherche =martel

8. Nous donnons ici les derniers chiffres officiels au plan national. Ils indiquent les grandes tendances.

9. Sur ces années pionnières, on pourra se référer à deux thèses récentes : Patrick COUFFIN, Cinquante ans d'occitan dans l'enseignement public, entre légitimité et légalité (L'enseignement du Languedocien: à l'école, au collège et au Lycée). 682 p. Thèse : Études Oc-citanes: Montpellier III, janvier 2008. Yan LESPOUX, Des années 1940 aux années 1990, un demi-siècle de revendication en faveur de l'enseignement de la langue d'oc, thèse, Pau, 8 septembre 2009.

10. Cependant, cette fréquentation est encouragée par de nombreux projets de collectage, qui mettent en rapport les nouveaux apprenants et les locuteurs naturels, détenteurs d'un savoir linguistique d'une qualité inégalée.

11. Charles CAMPROUX (1908-1994), agrégé de lettres, professeur de lycée, puis chargé de la chaire de langue et littérature d'oc à la faculté de lettres de l'IEO. Écrivain, linguiste, toponymiste, historien de la littérature, spécialiste des troubadours.

12. Robert LAFONT (1923-2009), professeur en lycée puis à l'Université. Immense intellectuel polygraphe (102 volumes publiés, ainsi que des milliers d'articles. Écrivain, linguiste, sociolinguiste, historien, historien de la littérature, animateur d'équipes de recherches, militant engagé. On trouvera une bibliographie sélective sur http://www.felco-creo.org/mdoc/ detail_fr.php ?categ =sitbibli\&id $=210$ et une présentation de l'écrivain sur : http://www.felcocreo.org $/$ mdoc/detail_fr.php ?categ =fac\&id $=254$

13. http://www.cndp.fr/Produits/DetailSimp.asp ?Id =81204

14. http://www.sceren.com/cyber-librairie-cndp.asp ?l =cant-de-las-vilas\&prod =14842

15. Chercheurs d'Oc, à la découverte d'un espace, d'une langue, d'une culture, Toulouse, CRDP de MidiPyrénées, 2004. ISBN : 2-86565-020-0

16. "L'Université, instrument de sauvetage de l'occitan?", en collaboration avec Carmen AlénGarabato, in L'école, instrument de sauvetage des langues menacées, travaux réunis par Chrystelle Burban et Christian Lagarde, Actes du colloque du 30 septembre et 1er octobre 2005, Perpignan, CRILAUP, Presses Universitaires de Perpignan, collection Études. 


\section{RÉSUMÉS}

La réalité de l'enseignement de l'occitan est très mouvante dans un espace linguistique très vaste. La créativité de la langue et la culture occitanes est à la fois très ancienne et très dynamique. Ce prestige culturel ainsi que la réflexion pédagogique et didactique essaient de contrebalancer un cadre juridique insuffisant et des blocages administratifs de toutes sortes.

The teaching of Occitan presents different aspects as it is spread over a very wide linguistic area. The creativity of the language and of the Occitan culture is both very old and very dynamic. This cultural prestige as well as pedagogic and didactic consideration try to compensate an inadequate legal framework and various administrative blockages.

\section{INDEX}

Mots-clés : contexte, enseignement, espace linguistique occitan, formation des maîtres, ressource pédagogique, texte

Keywords : context, occitan linguistic area, pedagogic resource, teacher training, teaching, text

\section{AUTEUR}

\section{MARIE-JEANNE VERNY}

Maître de conférences Occitan, Université Paul Valéry, secrétaire de la Fédération des Enseignants de Langue et Culture d'Oc (FELCO) 